\title{
Inducible nitric oxide synthase during the late phase of sepsis is associated with hypothermia and immune cell migration
}

\author{
Yudai Takatani ${ }^{1} \cdot$ Kenji Ono $\mathbb{C}^{2} \cdot$ Hiromi Suzuki ${ }^{2} \cdot$ Masato Inaba $^{1} \cdot$ Makoto Sawada $^{2} \cdot$ Naoyuki Matsuda ${ }^{1}$
}

Received: 6 October 2017 / Revised: 21 November 2017 / Accepted: 4 December 2017 / Published online: 14 February 2018

(c) United States \& Canadian Academy of Pathology 2018

\begin{abstract}
Hypothermia is a significant sign of sepsis, which is associated with poor prognosis, but few mechanisms underlying the regulation of hypothermia are known. Inducible nitric oxide synthase (iNOS) is a key inflammatory mediator of sepsis. However, the therapeutic benefit of iNOS inhibition in sepsis is still controversial, and requires elucidation in an accurate model system. In this study, wild-type (WT) mice showed temperature drops in a biphasic manner at the early and late phase of sepsis, and all mice died within $48 \mathrm{~h}$ of sepsis. In contrast, iNOS-knockout (KO) mice never showed the second temperature drop and exhibited improved mortality. Plasma nitric oxide (NO) levels of WT mice increased in the late phase of sepsis and correlated to hypothermia. The results indicate that iNOS-derived NO during the late phase of sepsis caused vasodilation-induced hypothermia and a lethal hypodynamic state. The expression of the iNOS mRNA was high in the lung of WT mice with sepsis, which reflects the pathology of acute respiratory distress syndrome (ARDS). We obtained the results in a modified keyhole-type cecal ligation and puncture model of septic shock induced by minimally invasive surgery. In this accurate and reproducible model system, we transplanted the bone marrow cells of GFP transgenic mice into WT and iNOS-KO mice, and evaluated the role of increased pulmonary iNOS expression in cell migration during the late phase of sepsis. We also investigated the quantity and type of bone marrow-derived cells (BMDCs) in the lung. The number of BMDCs in the lung of iNOS-KO mice was less than that in the lung of WT mice. The major BMDCs populations were CD11b-positive, iNOS-negative cells in WT mice, and Gr-1-positive cells in iNOS-KO mice that expressed iNOS. These results suggest that sustained hypothermia may be a beneficial guide for future iNOS-targeted therapy of sepsis, and that iNOS modulated the migratory efficiency and cell type of BMDCs in septic ARDS.
\end{abstract}

\section{Introduction}

The definition of sepsis has recently changed from conventional "systemic inflammatory response to infection" to "life-threatening organ dysfunction caused by a dysregulated host response to infection" [1,2]. Therefore, we have to improve our understanding of the mechanism of organ dysfunction to develop an effective therapy for sepsis. Excessive production of nitric oxide (NO) from inducible

Naoyuki Matsuda

nmatsuda@med.nagoya-u.ac.jp

1 Department of Emergency and Critical Care Medicine, Nagoya University Graduate School of Medicine, Aichi 466-8560, Japan

2 Division of Stress Adaptation and Protection, Department of Brain Function, Research Institute of Environmental Medicine, Nagoya University, Aichi 464-8601, Japan nitric oxide synthase (iNOS) worsens systemic hypotension and organ dysfunction in sepsis [3]. However, some animal studies of sepsis reported that inhibition of iNOS worsened mortality $[4,5]$, whereas others reported that inhibition improved mortality [6, 7]. Clinical studies of sepsis using selective iNOS inhibitors have not been designed yet. Thus, the therapeutic benefits of iNOS inhibition need to be evaluated in sepsis and septic shock.

Alteration of body temperature is one of the most frequent symptoms in sepsis. Recently, several studies have shown that hypothermia is associated with organ dysfunction and causes high mortality [8, 9]. However, few mechanisms underlying the regulation of hypothermia in sepsis are known. Several animal studies have suggested that iNOS is involved in hypothermia, and iNOS-knockout (KO) mice have improved hypothermia in a lipopolysaccharide (LPS)-induced sepsis model [10]. The authors suggest that excess NO production from iNOS leads to mitochondrial dysfunction, decreased ATP production, and 
decreased thermogenesis. However, another study reported that iNOS-KO mice showed worse hypothermia in LPSinduced sepsis [11]. They suggest a neuroendocrine mechanism in which central NO produced by iNOS inhibits the release of the endogeneous antipyretic vasopressin. That is, the role of iNOS-derived NO in thermoregulation during septic shock is still controversial, and should be elucidated in a more accurate model system.

Furthermore, the role of iNOS-derived NO may vary depending on the organ and cell type [12]. Acute respiratory distress syndrome (ARDS) is a representative pathology of organ dysfunction accompanied by septic shock, and is characterized by high morbidity and mortality [13, 14]. Many studies have shown that iNOS and NO increased in septic ARDS [14], but the cell type-specific roles of iNOS have not been fully elucidated. Several studies have generated reciprocal bone marrow transplanted iNOS chimeric mice, and examined iNOS activity in two separate pulmonary populations: bone marrow-derived inflammatory cells, such as macrophages and neutrophils, and pulmonary parenchymal cells, such as epithelial, smooth muscle, and endothelial cells [14-17]. Most of the increased NO production in septic ARDS was due to parenchymal cells [17], whereas pulmonary microvascular protein leak and oxidant stress were due to bone marrow-derived inflammatory cells $[15,16]$. However, these studies have not examined immature bone marrow-derived cells (BMDCs). In particular, the roles of BMDCs such as endothelial progenitor cells $[18,19]$ and mesenchymal stem cells [20], which have protective roles in septic ARDS, remain unclear.

A standard animal model of sepsis can be induced by cecal ligation and puncture (CLP) [21]. In the CLP model, it is possible to detect various inflammatory mediators and reproduce clinical pathology related to sepsis, such as shock, body temperature changes, and multiple organ failure [22]. In the CLP model, the severity of sepsis varies according to several surgical procedures, such as the length of ligation, needle size, and number of punctures. To stabilize the severity and enhance reproducibility, we modified and improved the CLP model, which is characterized by minimally invasive keyhole surgery in the abdomen [23, 24]. In this model, we evaluated the temperature changes and mortality of wild-type (WT) mice and compared them with that of iNOS-KO mice. We also determined the expression of iNOS mRNA in various organs. Furthermore, to assess the role of iNOS in cell migration in sepsis, we performed a bone marrow transplantation (BMT) experiment as previously described $[25,26]$, which enabled us to distinguish BMDCs from tissue-resident cells. Our results suggest that iNOS is associated with hypothermia and immune cell migration of BMDCs in the lung in the late phase of sepsis.

\section{Materials and methods}

\section{Animals}

Male C57BL/6 mice (8-12 weeks old, Nihon Charles River, Tokyo, Japan), male iNOS-KO mice (8-12 weeks old, B6.129P2-Nos $2<\mathrm{tmILau}>/ \mathrm{J}$, The Jackson Laboratories, ME, USA), and GFP transgenic male mice $(\operatorname{TgN}(\beta$-actEGFP)Osb) [27] were used. Mice were housed in a room maintained at $22-24{ }^{\circ} \mathrm{C}$ on a $12 \mathrm{~h}$ light $/ 12 \mathrm{~h}$ dark cycle. They were provided with food (MF pellet, Oriental Yeast Co. Ltd., Tokyo, Japan) and water ad libitum. All studies were performed in accordance with the Nagoya University Guide for the Care and Use of Animals and were approved by the Nagoya University Care and Use Committee.

\section{Keyhole cecal ligation and puncture}

We performed the "keyhole-type CLP surgery", in which the skin incision area is reduced to $5 \mathrm{~mm}$, according to a previously described protocol $[23,24]$ with a few modifications. After mice was anesthetized with isoflurane, a $5 \mathrm{~mm}$ paramedian incision was made through the skin and peritoneum of the left abdomen to expose the cecum. The cecum was ligated with 4-0 nylon $5 \mathrm{~mm}$ from the cecal tip and punctured twice with a 21-gauge needle. After the operation, the abdomen was closed and the exposed skin due to the incision was covered with the surrounding abdominal fur. We subsequently injected $1 \mathrm{~mL}$ of prewarmed $0.9 \%$ saline solution subcutaneously with a 25-gauge needle for fluid resuscitation, and mice were put on a heating pad (BWT-100A, Bio Research Center, Nagoya, Japan) until they recovered from the anesthesia and the temperature returned to that before the operation. During sham operation, the cecum was exposed but ligation and puncture were not performed.

\section{Measurement of body temperature}

We measured body temperature of all mice involved using a rectal temperature sensor (BWT-100A, Bio Research Center, Nagoya, Japan) without anesthesia. Measurement occurred just before and after the surgical procedure, and 6 $\mathrm{h}$ and $24 \mathrm{~h}$ after the operation. The temperature of three C57BL/6 mice and three iNOS-KO mice were monitored every $1 \mathrm{~h}$ for 2 days after the operation.

\section{Administration of a selective iNOS inhibitor}

$\mathrm{N}$-(3-(aminomethyl) benzyl) acetamidine $(1400 \mathrm{~W})$, a selective iNOS inhibitor (Wako Pure Chemical, Osaka, Japan), was dissolved in $0.9 \%$ saline before each 
experiment and injected at a dose of $0.5 \mathrm{mg} / \mathrm{kg}$ into the tail vein during CLP and $6 \mathrm{~h}$ later.

\section{Survival analysis}

To analyze the survival rate, mice were monitored for 7 days. WT mice subjected to CLP (WT CLP, $n=11$ ), iNOS-KO mice subjected to CLP (iNOS-KO CLP, $n=9$ ), WT mice subjected CLP with treatment of $1400 \mathrm{~W}$ (WT $1400 \mathrm{~W}$ CLP, $n=10$ ), WT mice that received 5-fluorouracil (5-FU) and underwent CLP without BMT (WT 5-FU CLP, $n=5$ ), WT mice subjected to CLP after receiving 5-FU and undergoing BMT (WT BMT CLP, $n=9$ ), iNOS-KO mice subjected to CLP and undergoing BMT (iNOS-KO BMT CLP, $n=7$ ) were used for analysis.

\section{NO measurement}

Mice receiving isoflurane anesthesia were killed and blood samples were obtained by cardiac puncture $6 \mathrm{~h}$ or $24 \mathrm{~h}$ after the operation. Mice were perfused intracardially with $0.1 \mathrm{M}$ phosphate-buffered saline (PBS); organs were removed, embedded in Tissue-Tek OCT compound (Sakura Finetek Japan Co. Ltd., Tokyo, Japan), and stored at $-80^{\circ} \mathrm{C}$ for biochemical or histological analyses. Heparinized plasma samples were centrifuged at $7000 \times g$ for $20 \mathrm{~min}$ at $4{ }^{\circ} \mathrm{C}$ with Amicon Ultra-4 (10 kDa cut off) centrifugal filter unit (Millipore Corporation, MA, USA) for deproteinization. Plasma nitrate and nitrite levels were measured by a fluorescence method $\left(\mathrm{NO}_{2} / \mathrm{NO}_{3}\right.$ Assay kitCII(Colorimetric), Dojindo Laboratories, Kumamoto, Japan).

\section{RNA extraction and real-time PCR}

Total RNA was extracted from frozen tissue sections of the brain, lung, liver, kidney of WT mice at $6 \mathrm{~h}$ or $24 \mathrm{~h}$ after the operation ( $n=3$, respectively). RNA extraction and cDNA synthesis were performed as described previously [28]. cDNA was amplified and analyzed with Power SYBR Green PCR Master Mix and QuantStudio 12K Flex Real-Time PCR System (Applied Biosystems, Tokyo, Japan). The threshold cycle (CT) values for iNOS were normalized to that of glyceraldehyde-3-phosphate dehydrogenase (GAPDH). The mean value of brain samples of WT mice that were killed $6 \mathrm{~h}$ after sham operation was set to 1 . Primers used were as follows: iNOS (sense primer: GCAGCTACTGGGTCAAAGACAA, antisense primer: TCTCTGCCTATCCGTCTCGTC), GAPDH (sense primer: TGCACCACCAACTGCTTAG, antisense primer: GATGCAGGGATGATGTTC).

\section{Bone marrow transplantation}

Many experimental BMT studies have used an irradiation protocol $[14,19]$. However, tissue damage and inflammation from irradiation accelerates cell turnover, which could modify the course of sepsis and the population of immunocompetent cells [29]. To exclude the side effects of irradiation, we adopted a minimally invasive protocol, as previously described [25, 26], that uses dose de-escalation of 5-FU, which reduces the number of recipient hematopoietic cells. GFP transgenic mice were used as donors, and WT mice and iNOS-KO mice were used as recipients. Twelve days after BMT, one drop of blood from the tail vein of recipient mice receiving isoflurane anesthesia was taken for chimerism analysis. Chimerism was confirmed using a Tali ${ }^{\mathrm{TM}}$ Image-Based Cytometer (Thermo Fisher Scientific). The percentage of GFP-positive cells of 6-12 $\mu \mathrm{m}$ in total cells was calculated. Thirteen days after BMT, chimeric mice underwent sham or CLP operation.

\section{Immunohistochemical analysis}

Chimeric mice were killed $24 \mathrm{~h}$ after the sham or CLP operation, perfused with $0.1 \mathrm{M}$ PBS, and the lungs isolated for immunohistochemical analysis. Frozen lung sections (10 $\mu \mathrm{m})$ were cut using a cryostat microtome (Leica Microsystems K.K., Tokyo, Japan), transferred to MAS-coated slides (Matsunami Glass Ind., Ltd., Japan), and air dried. Sections were fixed with $4 \%$ paraformaldehyde in PBS at $4{ }^{\circ}$ $\mathrm{C}$ for $10 \mathrm{~min}$ and treated with $0.1 \%$ triton-X for $10 \mathrm{~min}$ to improve permeability. Double-labeled sections were stained with rabbit polyclonal antibodies against GFP (598; MBL, Nagoya, Japan) at a dilution of 1:500 and with rat monoclonal antibodies against CD11b (70-0112; TONBO biosciences, San Diego, CA), Gr-1 (553123; BD Biosciences, Japan), or ER-MP12 (T-2001; BMA, Switzerland) at a dilution of 1:200. The reaction was visualized with fluorescein isothiocyanate (FITC)-conjugated goat Fab'2 anti-rabbit $\mathrm{IgG}$ (L43001; Caltag) at a dilution of 1:250 and Alexa546conjugated anti-rat IgG (A-11081; Thermo Fisher Scientific) at a dilution of 1:500, and photographed using a fluorescent microscope (BZ-X700, Keyence Co., Osaka, Japan). The nucleus was stained using Hoechst 33342 (Thermo Fisher Scientific). Triple-labeled sections were stained with mouse monoclonal antibodies against iNOS (610328; BD Biosciences, Japan) at a dilution of 1:250, rabbit polyclonal antibodies against GFP at a dilution of 1:500, and rat monoclonal antibodies against either CD11b, Gr-1, or ERMP12 at a dilution of 1:200. The reaction was visualized with Alexa546-conjugated goat anti-mouse IgG (A21045; Life Technologies) at a dilution of 1:500, FITC-conjugated goat anti-rabbit IgG at a dilution of 1:250, and Alexa350- 
conjugated goat anti-rat IgG (A-21093; Thermo Fisher Scientific) at a dilution of 1:250. Cells in the image were counted at $\times 200$ magnification. To evaluate the percentage of GFPpositive cells, at least $100 \mathrm{GFP}$-positive cells in total were captured. For analysis of cell number, the number of positive cells in each field was counted manually and an average was calculated from five randomly chosen fields.

\section{Statistical analysis}

Values were expressed as mean \pm SEM. Statistical analyses were performed using two-tailed Student's $t$-test for twogroup comparisons, or one-way ANOVA followed by Tukey's test for multiple-group comparisons. Pearson correlation analysis was used to evaluate the correlation between NO concentration and changes in rectal temperature. Kaplan-Meier survival curves were compared by a logrank test. $P<0.05$ was considered statistically significant. All statistical analyses were performed using EZR software (Jichi Medical University, Saitama, Japan), which is a graphical user interface for R (The R Foundation for Statistical Computing, Vienna, Austria) [30].

\section{Results}

\section{Inhibition of iNOS-improved hypothermia and mortality}

WT mice and iNOS-KO mice underwent CLP to induce sepsis. They were monitored for 7 days after CLP for

A

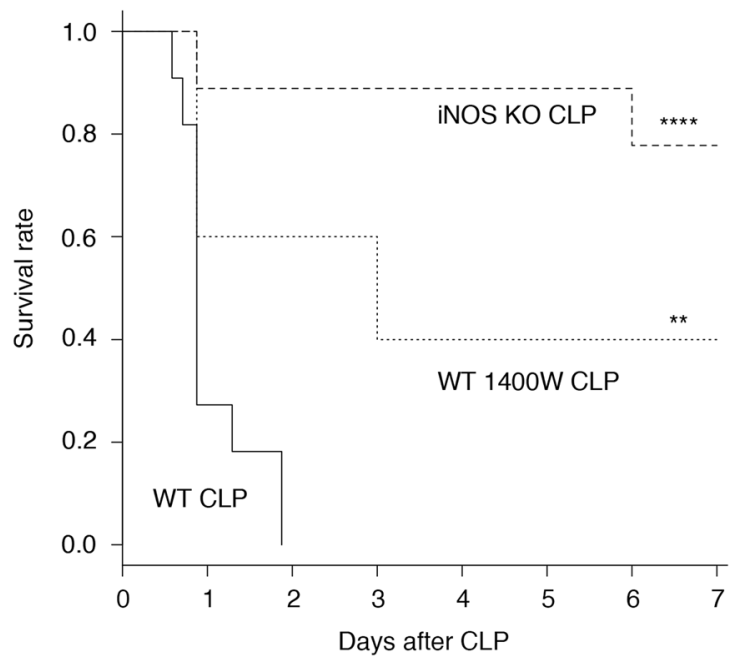

survival analysis (Fig. 1a) and body temperature (Fig. 2a-d). All WT CLP group $(n=11)$ died within $48 \mathrm{~h}$, but $\sim 78 \%$ of iNOS-KO CLP group $(n=9)$ survived more than 7 days after CLP. When WT mice were injected intravenously with a selective iNOS inhibitor $1400 \mathrm{~W}(0.5$ $\mathrm{mg} / \mathrm{kg}$ ) at the time of CLP and $6 \mathrm{~h}$ later (WT $1400 \mathrm{~W}$ CLP, $n=10$ ), $40 \%$ mice survived for more than 7 days. These results show that the complete or drug-induced inhibition of iNOS-improved mortality $(P<0.0001, \quad P=0.00359$, respectively). All WT mice and iNOS-KO mice subjected to sham operation survived more than 7 days (data not shown).

For the analysis of body temperature, we measured the rectal temperature of the WT CLP $(n=11)$ and the iNOSKO CLP group ( $n=9) 6 \mathrm{~h}$ after CLP, and that of surviving mice $24 \mathrm{~h}$ after CLP (WT CLP: $n=3$, iNOS-KO CLP: $n=$ 8 ). Before the analyses, three mice were randomly selected from each group and temperature was measured every $1 \mathrm{~h}$ until $48 \mathrm{~h}$ after CLP (Fig. 2a). In both groups, a decrease in body temperature $\sim 3 \mathrm{~h}$ after CLP and a subsequent transient state of equilibrium for $\sim 3 \mathrm{~h}$ were observed. Afterwards, a progressive decrease in temperature occurred, with or without a transient recovery phase, and then death in the WT CLP group. In contrast, temperature increased and recovered to the initial temperature in the iNOS-KO CLP group. The 20 mice (WT CLP: $n=11$, iNOS-KO CLP: $n=9)$ were divided into two groups: the survive group that survived more than 7 days after CLP, and the nonsurvive group that died within 7 days (Fig. $2 b-d$ ). There was no significant difference in the temperature decrease between the two groups $6 \mathrm{~h}$ after CLP (survive group: 2.96 $\pm 0.42{ }^{\circ} \mathrm{C}$; non-survive group: $\left.4.23 \pm 0.58^{\circ} \mathrm{C}, P=0.1508\right)$

B

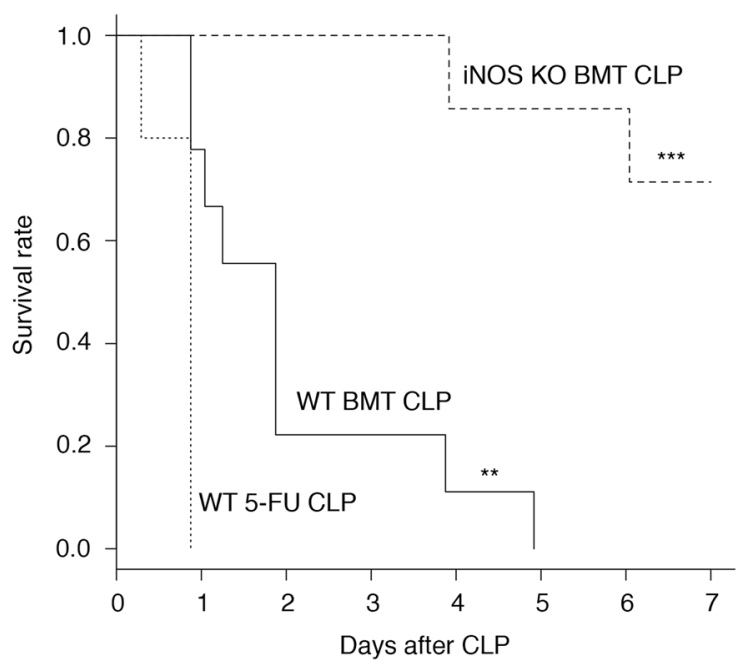

Fig. 1 Complete or drug-induced inhibition of iNOS and the mortality. a Survival of WT mice subjected to CLP (WT CLP, $n=11$ ), iNOS-KO mice subjected to CLP (iNOS-KO CLP, $n=9$ ), and WT mice subjected to CLP with treatment of a selective iNOS inhibitor, $1400 \mathrm{~W}(0.5 \mathrm{mg} / \mathrm{kg}$ ) (WT $1400 \mathrm{~W}$ CLP, $n=10$ ). $1400 \mathrm{~W}$ was administered twice- at the time of CLP and $6 \mathrm{~h}$ later. $* * P<0.01$, $* * * P<0.0001$, compared with WT CLP (log-rank test). b Survival of WT mice that received 5-FU and underwent CLP without BMT (WT 5-FU CLP, $n=5$ ), WT mice subjected to CLP after receiving 5-FU and undergoing BMT (WT BMT CLP, $n=9$ ), iNOS-KO mice subjected to CLP and undergoing BMT (iNOS-KO BMT CLP, $n=7$ ). ${ }^{* *} P<0.01$, ${ }^{* * *} P<0.001$, compared with WT 5-FU CLP (log-rank test) 

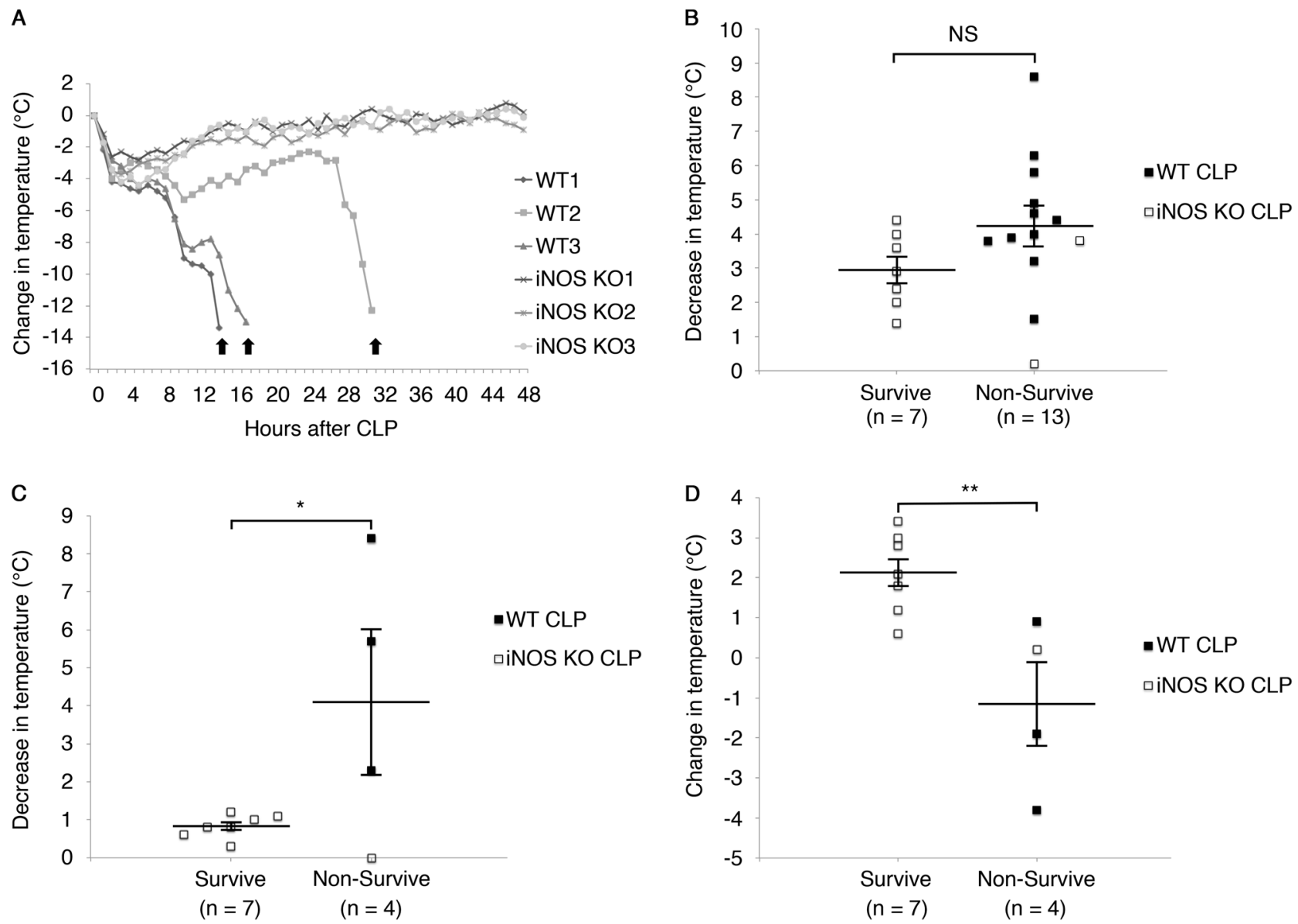

Fig. 2 iNOS-KO mice attenuated hypothermia in the late phase of sepsis and improved mortality after CLP. a Time course of hypothermia in WT mice (WT 1, WT 2, WT 3) and iNOS-KO mice (iNOS-KO 1, iNOS-KO 2, iNOS-KO 3) after CLP. Arrows represent death. b Correlation between survival and rectal temperature $6 \mathrm{~h}$ after CLP in WT mice $(n=11)$ and iNOS-KO mice $(n=9)$. These 20 mice were divided into two groups: the survive group $(n=7)$ that survived more than 7 days after CLP, and the non-survive group $(n=13)$ that died within 7 days. Body temperatures were compared between the two groups. c Correlation between survival and rectal temperature $24 \mathrm{~h}$ after CLP in WT mice $(n=3)$ and iNOS-KO mice $(n$ $=8)$. These 11 mice are divided into the survive group $(n=7)$ and the non-survive group $(n=4)$, and the temperatures were compared. $\mathbf{d}$ Correlation between survival and changes in rectal temperature from $6 \mathrm{~h}$ to $24 \mathrm{~h}$ after CLP in WT mice $(n=3)$ and iNOS-KO mice $(n=8)$. These 11 mice were divided into the survive group $(n=7)$ and the non-survive group $(n=4)$, and the temperatures were compared. $* P<0.05$, $* * P<0.01$, NS not significant, two-tailed $t$-test. Error bars represent the mean \pm SEM

(Fig. 2b). The decrease in temperature $24 \mathrm{~h}$ after CLP was significantly attenuated in the survive group, compared with that in the non-survive group $\left(0.83 \pm 0.12^{\circ} \mathrm{C} ; 4.10 \pm 1.85^{\circ}\right.$ $\mathrm{C}$, respectively, $P=0.0383$ ) (Fig. 2c). There was recovery from hypothermia from $6 \mathrm{~h}$ to $24 \mathrm{~h}$ after CLP in the survive group, but not in the non-survive group $\left(2.13 \pm 0.38^{\circ} \mathrm{C}\right.$; $-1.15 \pm 1.06{ }^{\circ} \mathrm{C}$, respectively, $P=0.0064$ ) (Fig. $2 \mathrm{~d}$ ).

Next, we evaluated NO production. WT mice and iNOSKO mice underwent CLP or sham operation, and were killed $6 \mathrm{~h}$ or $24 \mathrm{~h}$ after CLP ( $n=3$ or 4 , respectively). Plasma nitrate and nitrite levels were measured to indicate NO production (Fig. 3a). In the WT CLP group, levels were significantly higher than the sham group $24 \mathrm{~h}$ after CLP $(182.90 \mu \mathrm{M}, 59.34 \mu \mathrm{M}$, respectively, $P<0.0001)$, but not 6 h after CLP $(105.55 \mu \mathrm{M}, 62.65 \mu \mathrm{M}, P=0.228)$. In the WT
$1400 \mathrm{~W}$ CLP group, levels $24 \mathrm{~h}$ after CLP significantly decreased $(96.75 \mu \mathrm{M})$ compared with the WT CLP group $(P=0.00018)$. In the iNOS-KO groups with confirmed disruption of the iNOS gene, there were no significant increases in NO levels. When the correlation between plasma nitrite/nitrate levels and the temperature of WT CLP or iNOS-KO CLP groups was analyzed, there was a strong correlation at $6 \mathrm{~h}(r=0.732, P=0.039)$ (Fig. $3 \mathrm{~b})$ and $24 \mathrm{~h}$ $(r=0.702, P=0.052)$ (Fig. 3c) after CLP.

\section{iNOS expression in the lung increased continuously}

To understand iNOS induction by CLP, we determined iNOS mRNA levels in the brain, lung, liver, and kidney of WT mice subjected to sham or CLP operation ( $n=3$ for both) via 

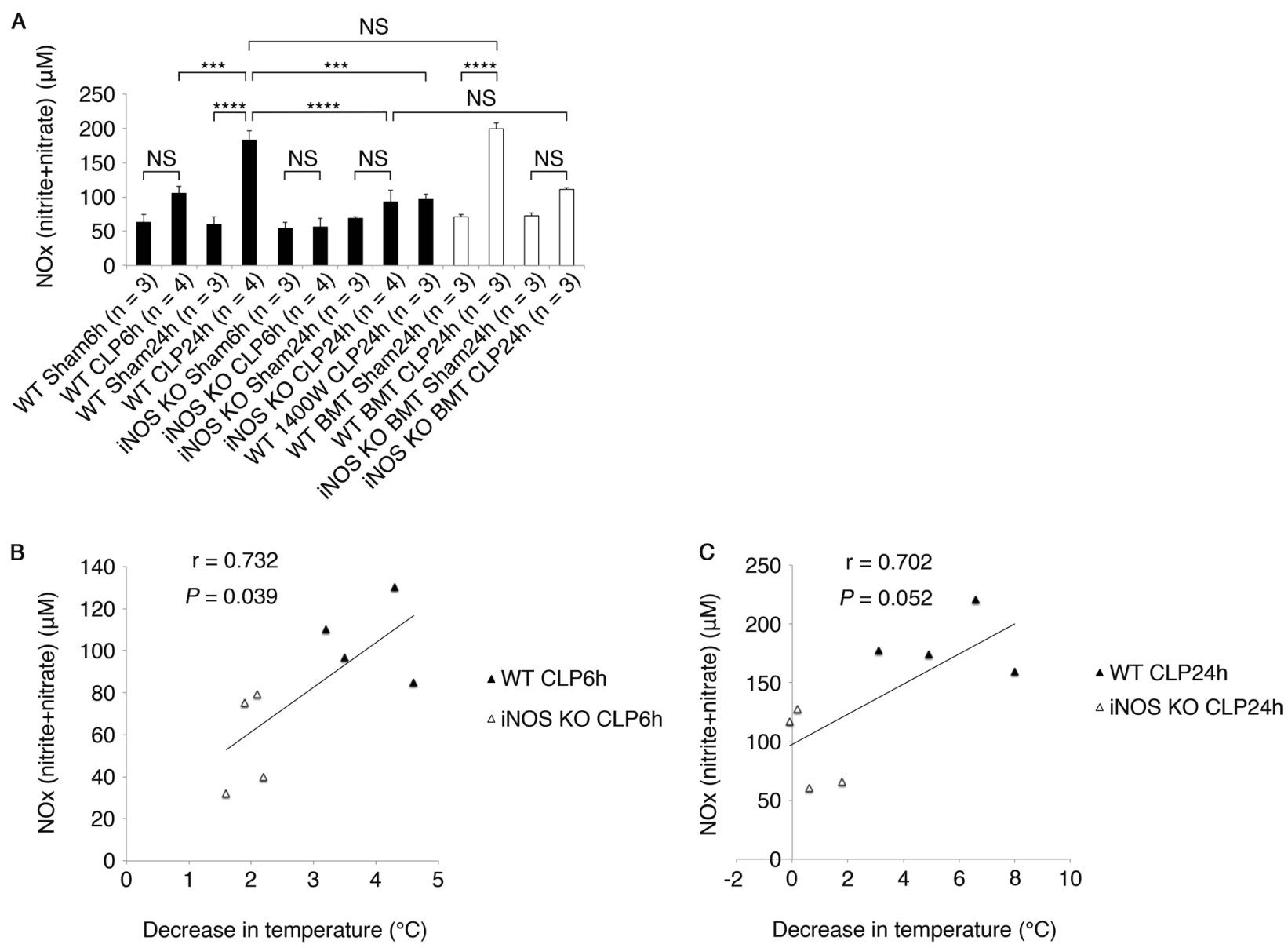

Fig. 3 Plasma nitrite/nitrate levels were related to decrease in temperature. a Comparison of plasma nitrite/nitrate levels in WT and iNOS-KO mice: WT mice subjected to sham/CLP and killed $6 \mathrm{~h}$ or $24 \mathrm{~h}$ after the operation (WT sham $6 \mathrm{~h}$, WT CLP $6 \mathrm{~h}$, WT sham $24 \mathrm{~h}$, WT CLP $24 \mathrm{~h}$ ); iNOS-KO mice subjected to sham/CLP and killed $6 \mathrm{~h}$ or $24 \mathrm{~h}$ after the operation (iNOS-KO sham $6 \mathrm{~h}$, iNOS-KO CLP $6 \mathrm{~h}$, iNOS-KO sham $24 \mathrm{~h}$, iNOS-KO CLP $24 \mathrm{~h}$ ); WT mice subjected to CLP with treatment of a selective iNOS inhibitor, $1400 \mathrm{~W}$ (WT $1400 \mathrm{~W}$ CLP $24 \mathrm{~h}$ ); WT mice subjected to sham/CLP after BMT and killed $24 \mathrm{~h}$ after the operation (WT BMT sham $24 \mathrm{~h}$, WT BMT CLP $24 \mathrm{~h}$ ); iNOS-KO mice subjected to sham/CLP after BMT and killed $24 \mathrm{~h}$ after the operation (iNOS-KO BMT sham $24 \mathrm{~h}$, iNOS-KO BMT CLP $24 \mathrm{~h}$ ). $n=3$ to 4 . Error bars represent the mean \pm SEM. $* * P<0.01$, $* * * P<0.001$, $* * * * P<0.0001$, NS not significant (one-way ANOVA followed by Tukey's test). b, c Correlation between plasma nitrite/nitrate levels and rectal temperature $6 \mathrm{~h}(\mathbf{b})$ and $24 \mathrm{~h}$ (c) after CLP. $n=4 . r$ correlation coefficient (Pearson correlation analysis)

quantitative real-time PCR (Fig. 4). The expression level of iNOS mRNA in the lung increased both $6 \mathrm{~h}$ and $24 \mathrm{~h}$ after CLP $(P=0.0039, P=0.0402$, respectively), but there were no significant increases in the other three organs.

\section{iNOS modulated the migratory efficiency and cell type of BMDCs in septic ARDS}

In our improved CLP model, we confirmed that septic shock induced overproduction of NO from iNOS, especially in the lung. On the basis of these results, we performed a BMT experiment to elucidate the role of BMDCs in septic ARDS. We transplanted bone marrow cells of GFP transgenic mice into WT mice and iNOS-KO mice, in which 5 -FU had been administered in advance, and performed CLP or sham operation. The percentage of GFP-positive cells in peripheral blood cells was not significantly different between WT and iNOS-KO mice: $13.3 \pm 2.3 \%$, WT mice $(n=13): 14.4 \pm 1.2 \%$, iNOS-KO mice $(n=13)$ $(P=0.6619)$.

As shown in Fig. 1b, survival was evaluated after CLP. All WT mice that received 5-FU but did not undergo BMT died within $24 \mathrm{~h}$ after CLP (WT 5-FU CLP, $n=5$ ). On the other hand, two WT mice that received BMT survived more than $48 \mathrm{~h}$ after CLP (WT BMT CLP, $n=9$ ), and five iNOS-KO mice that received BMT survived more than 7 days (iNOS-KO BMT CLP, $n=7$ ). The WT BMT CLP and iNOS-KO BMT CLP groups had significantly improved mortality compared with the WT 5-FU CLP group $(P=0.00462, P=0.000918$, respectively $)$. The mortality of the WT BMT CLP group significantly improved compared with the WT CLP group $(P=0.0332)$, 


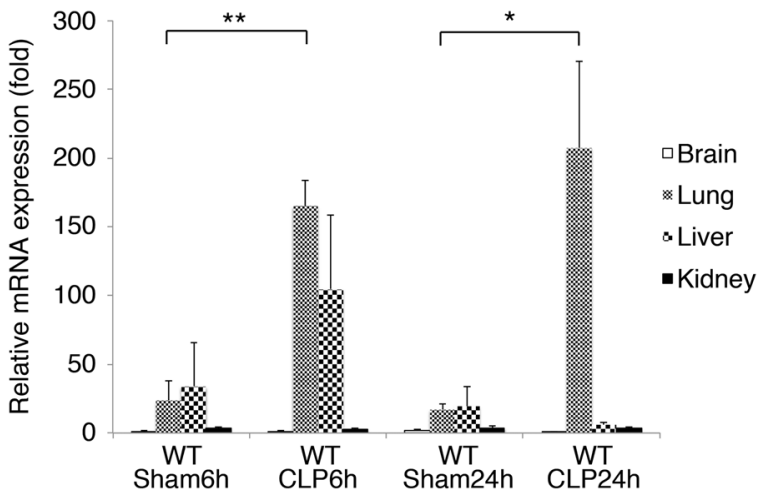

Fig. 4 iNOS gene expression in the early and late phase of sepsis. iNOS mRNA levels in the brain, lung, liver, and kidney of WT mice via quantitative real-time PCR $(n=3)$. WT sham $6 \mathrm{~h}$ : WT mice killed $6 \mathrm{~h}$ after sham operation, WT CLP $6 \mathrm{~h}$ : WT mice killed $6 \mathrm{~h}$ after CLP operation, WT sham $24 \mathrm{~h}$ : WT mice killed $24 \mathrm{~h}$ after sham operation, WT CLP $24 \mathrm{~h}$ : WT mice killed $24 \mathrm{~h}$ after CLP. Error bars represent the mean \pm SEM. The CT values for iNOS were normalized to that of GAPDH. The mean value of brain samples of WT sham $6 \mathrm{~h}$ was set to 1. $* P<0.05$, $* * P<0.01$, compared with sham group (two-tailed $t$-test)

but there was no significant difference in mortality between the iNOS-KO BMT CLP and the iNOS-KO CLP groups $(P$ $=0.836$ ) (Fig. 1a).

We subsequently measured NO production of the BMT groups $24 \mathrm{~h}$ after sham or CLP operation ( $n=3$ for both) (Fig. 3a). The plasma nitrite/nitrate levels of the WT BMT CLP group were significantly higher than the sham group (WT BMT sham) $(199.18 \mu \mathrm{M}, 71.02 \mu \mathrm{M}$, respectively, $P<$ $0.0001)$, but were not different from that of the WT CLP group $(P=0.996)$. The plasma nitrite/nitrate levels of the iNOS-KO BMT CLP group was not significantly different from that of the sham group (iNOS-KO BMT sham) $(110.91 \mu \mathrm{M}, 72.49 \mu \mathrm{M}$, respectively), and also not significantly different from the iNOS-KO CLP group.

Next, we analyzed by immunohistochemistry the lung tissue of chimeric mice $24 \mathrm{~h}$ after sham or CLP operation to evaluate the population of BMDCs that migrated to the lung. The distribution of BMDCs was evaluated by tracing GFP-positive cells (Fig. 5a). In the iNOS-KO BMT CLP group, the number of GFP-positive BMDCs that migrated to the lung did not increase significantly compared with the iNOS-KO BMT sham group $\left(7.41 \pm 0.89\right.$ cells $/ \mathrm{mm}^{2}, 5.05$ \pm 1.27 cells $\left./ \mathrm{mm}^{2}, P=0.929\right)$. On the other hand, in the WT BMT CLP group, the number of BMDCs increased significantly compared with the WT BMT sham group $\left(118.23 \pm 5.11\right.$ cells $/ \mathrm{mm}^{2}, \quad 10.61 \pm 1.52$ cells $/ \mathrm{mm}^{2}, \quad P<$ $0.0001)$, and also increased compared with the iNOS-KO BMT CLP group $(P<0.0001)$. Next, we evaluated the cell type of GFP-positive BMDCs (Fig. 5b). The major populations of BMDCs that increased after CLP were CD11bpositive cells, such as macrophages in the WT BMT CLP group, and Gr-1-positive cells, such as granulocytes in the iNOS-KO BMT CLP group. ER-MP12-positive cells, such as murine early stage myeloid precursor cells and vascular endothelial cells $[31,32]$, were barely detected. We also examined iNOS protein expression in GFP-positive BMDCs (Fig. 5c). In the WT BMT CLP group, a significantly larger proportion of double-positive GFP/CD11b cells was iNOS-negative compared with the iNOS-KO BMT CLP group (\% of iNOS-positive cells: $5.51 \pm 3.01 \%$, $38.75 \pm 9.39 \%$, respectively, $P=0.028$ ). There was no significant difference in the proportion of iNOS-positive cells in double-positive GFP/Gr-1 and GFP/ER-MP12 cells. In the WT BMT sham group and the iNOS-KO BMT sham group, there were no GFP-positive BMDCs that expressed iNOS (data not shown).

\section{Discussion}

In this study, we demonstrated that the inhibition of iNOSimproved hypothermia in the late phase of sepsis and mortality in a modified keyhole-type CLP mouse model, where all WT mice show hypothermia and die within $48 \mathrm{~h}$. We have previously shown that the suppression of transcriptional factor nuclear factor- $\kappa \mathrm{B}$ activation, which was associated with the transcription of iNOS, improved pulmonary oxygenation [24,33] and mortality [33] in a CLP or LPS model of septic shock. These results are compatible with that of the present study, indicating that they are reproducible and reliable.

Hypothermia is a significant sign in sepsis [8,9], but little is known about its mechanism. We hypothesized that overproduction of NO derived from iNOS during the late phase of sepsis induced vasodilation, which then caused persistent heat loss. To examine this possibility, we monitored the rectal temperature of mice every $1 \mathrm{~h}$ after CLP (Fig. 2a). However, isoflurane anesthesia may result in the reduction of the initial temperature. To exclude the influence of anesthesia, we kept mice on a heating pad after the operation until they could maintain their original temperature and awaken from the anesthesia. Therefore, observed temperature changes after CLP was considered to result mainly from sepsis. In WT mice, temperature changes may be divided into three consecutive phases: phase of initial temperature drop, plateau phase, and phase of secondary temperature drop. Changes in the initial temperature drop and subsequent plateau phase, which represent the early phase of sepsis, were also observed in iNOS-KO mice. These changes resemble the three phases of development of core hypothermia during general anesthesia [34]: an initial decrease in temperature resulting from core-to-peripheral redistribution of body heat, then a slow and linear decrease caused by total body heat loss, and finally a temperature 

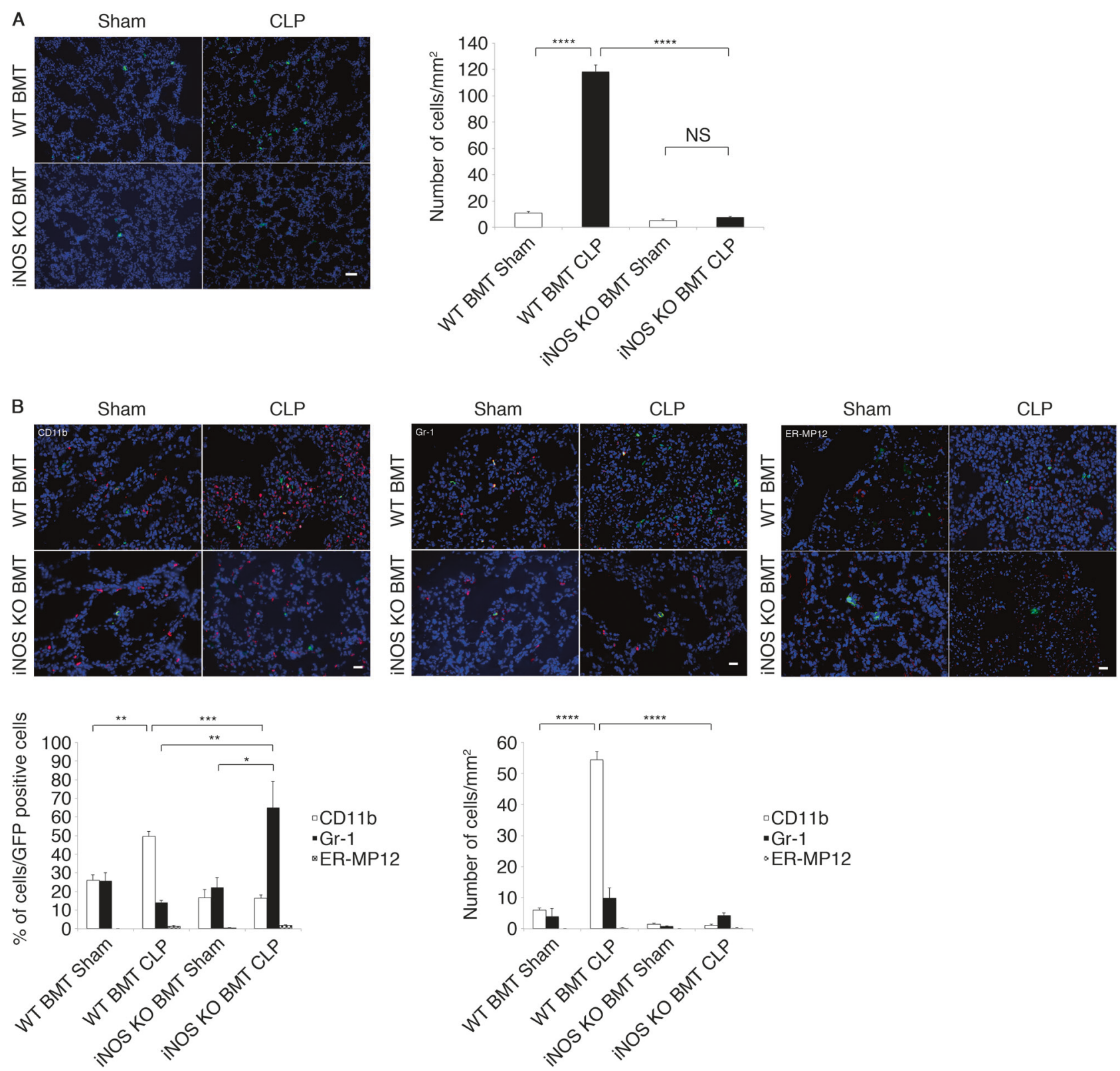

Fig. 5 Continued

plateau accompanied by metabolic heat production and vasoconstriction. Given that sepsis is one of the most common pathologies of distributive shock, the temperature drop in the early phase may result from the redistribution of body heat induced by several vasodilator mediators. Importantly, the second drop in temperature, which represents the late phase of sepsis, was never observed in iNOSKO mice, which indicates that iNOS has a key role in hypothermia in the late phase of sepsis. The contribution of iNOS in the decrease of core temperature in the late phase is supported by the increase in NO production in WT mice, not at $6 \mathrm{~h}$, but at $24 \mathrm{~h}$ after CLP (Fig. 3a). Changes in WT mice may reflect the classic two phases in sepsis: a hyperdynamic state in early sepsis, and a hypodynamic state in late sepsis [22]. That is, core hypothermia during hyperdynamic state could evolve into lethal hypothermia during hypodynamic state by the overproduction of NO via iNOS. This hypothesis agrees with the result that there was a correlation between poor outcomes and hypothermia during the late phase, but not the early phase (Fig. 2b-d). Temperature studies in human sepsis have shown that hypothermia in sepsis is a transient and self-limiting phenomenon, and is rarely observed in the moments preceding death [35]. Further analyses of sepsis-associated hypothermia are necessary to connect experimental and clinical evidence. 


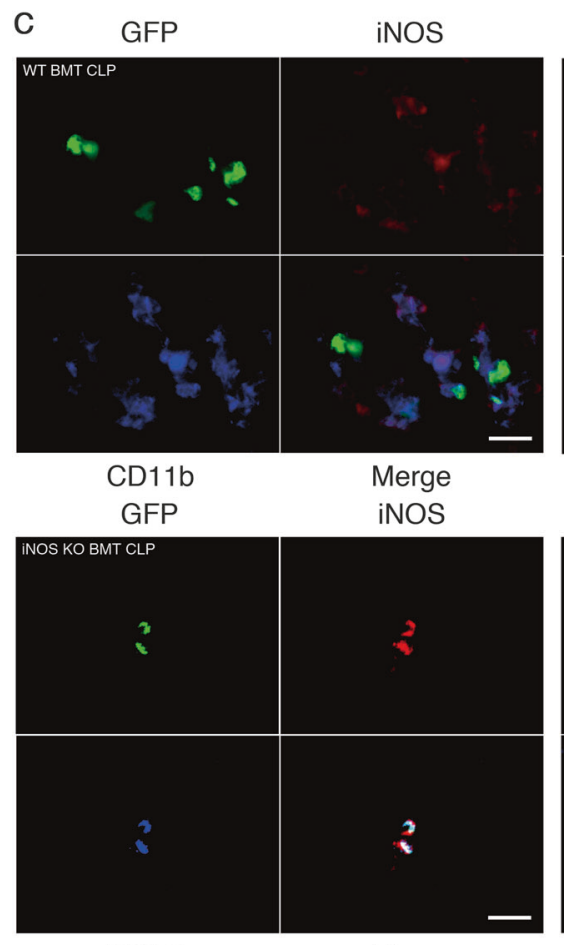

CD11b

Merge

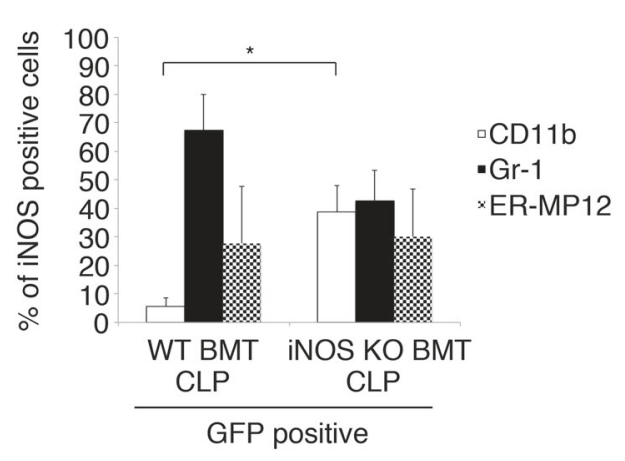

GFP

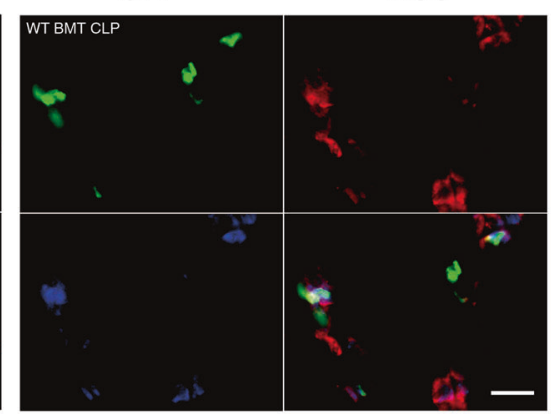

Gr-1

GFP

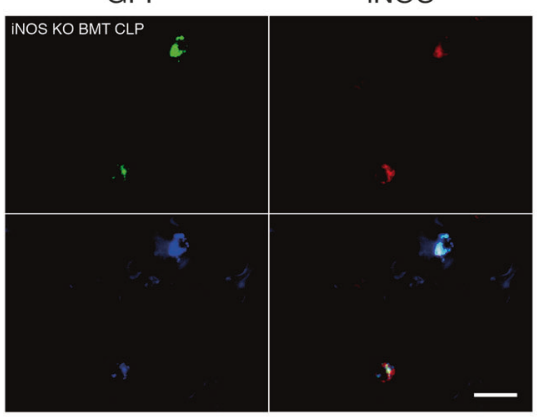

Gr-1

iNOS

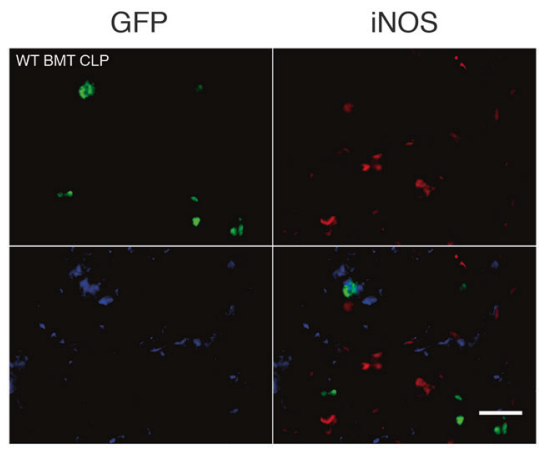

ER-MP12

GFP

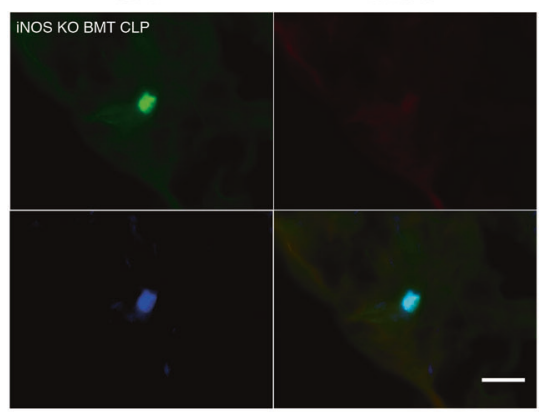

ER-MP12

Merge

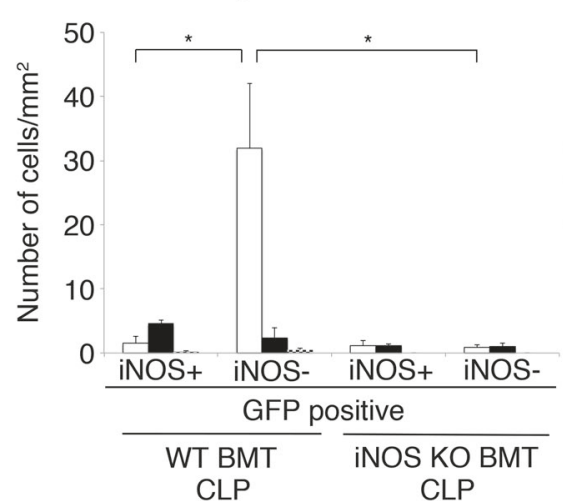

-CD11b

- Gr-1

-ER-MP12

Fig. 5 iNOS modulated the migratory efficiency and cell type of BMDCs in septic ARDS. a GFP-positive BMDCs migrated to the lung in WT and iNOS-KO chimeric mice subjected to sham/CLP after BMT and killed $24 \mathrm{~h}$ after the operation. Color staining: GFP, green. Nucleus is stained with Hoechst 33342. Scale bar, $50 \mu \mathrm{m}$. GFP-positive cells are counted (one-way ANOVA followed by Tukey's test). b Characteristics of GFP-positive BMDCs that migrated to the lung in WT and iNOS-KO chimeric mice. Color staining: GFP, green; CD11b, Gr-1, or ER-MP12, red. Nucleus is stained with Hoechst 33342. Scale bar, $20 \mu \mathrm{m}$. Double-positive GFP/CD11b, GFP/Gr-1, and GFP/ER-MP12 cells are counted (one-way ANOVA followed by Tukey's test). c Expression of iNOS in GFP-positive BMDCs that migrated to the lung in WT and iNOS-KO chimeric mice. Color staining: GFP, green; iNOS, red; CD11b, Gr-1, or ER-MP12, blue. Scale bar, $20 \mu \mathrm{m}$. GFP-positive cells are counted (two-tailed $t$-test for the comparison of the percentage of cells, one-way ANOVA followed by Tukey's test for the comparison of cell number). To evaluate the percentage of GFP-positive cells, at least 100 GFP-positive cells in total were counted. An average was calculated from cells counted in five fields chosen at random. Error bars represent the mean \pm SEM. $(n=3) * P<0.05, * * P<0.01, * * * P<0.001, * * * * P<0.0001$, NS not significant

Sustained iNOS gene expression after CLP was strongly observed in the lung (Fig. 4), which might relate to the pathology of ARDS [14]. The result that iNOS gene expression increased only in the lung when sepsis caused multiple organ dysfunction may be correlated with the results of previous studies showing that low levels of iNOS is expressed constitutively in the lung, especially in the type II alveolar cells, whereas iNOS is not expressed in the normal state in other organs [36, 37]. This may suggest that iNOS expression is easily induced in such pulmonary cells, which leads to the production of a large amount of NO in response to stimulation such as sepsis. In addition, the NO levels in blood significantly increased $24 \mathrm{~h}$ after CLP as shown in Fig. 3a. These results suggest that NO derived from lung cells is released in the bloodstream, which might cause multiple organ failure as a secondary effect. Thus, we 
considered the lung as a strong target of sepsis in our CLP model. To assess the contribution of migrating cells in septic ARDS, we performed BMT and examined the cellular source and role of iNOS in the lung. In WT mice, NO production in the WT BMT CLP group was not significantly different from that of the WT CLP group (Fig. 3a). Moreover, the major population of BMDCs in WT mice subjected to CLP was CD11b-positive cells, which is expressed in immunocompetent cells like macrophages, but most of these did not express iNOS (Fig. 5b, c). The results suggest that lung-resident cells were mainly responsible for sustained iNOS expression, consistent with previous reports [17]. Gr-1-positive cells, such as neutrophils, were a minor population. This result may be related to previous reports indicating that in severe sepsis, iNOS-derived NO reduced expression of CXCR2, $\beta 2$ integrins, and other adhesion molecules on the surface of neutrophils, inhibiting their migration [38, 39]. However, these results cannot explain the improvement in mortality in the WT BMT CLP group compared with the WT CLP group (Fig. 1a, b). Myeloid-derived suppressor cells, which express both CD11b and Gr-1, but were not identified in the present study, may have a protective role in sepsis [40-42]. Although a direct protective contribution from immature BMDCs was not confirmed in this study, several studies have reported that immature BMDCs attenuate inflammation in septic ARDS [18-20]. In addition to a diverse macrophage population [29], another newly identified population, called the segregated-nucleus-containing atypical monocyte, which has the characteristics of both monocytes and granulocytes, has been recently reported to be important for the development of bleomycin-induced lung fibrosis [43]. Thus, further analysis and identification of diverse populations of BMDCs are necessary for the pathologic clarification of septic ARDS.

In the iNOS-KO BMT CLP group, migration of GFPpositive BMDCs was significantly suppressed compared with that of the WT BMT CLP group (Fig. 5a, b). This suggests that iNOS-derived NO in the target organ may be the essential driving signal for BMDCs migration, or that inflammation of the lung was attenuated in iNOS-KO mice. The result is compatible with those of the report showing that NO induces the migration of CD34-positive cells such as bone marrow-derived progenitor cells via up-regulation of the expression of cell surface CXCR4 [44]. Moreover, this result may also explain no significant difference in survival between the iNOS-KO BMT CLP and the iNOSKO CLP groups (Fig. 1a, b). As we shown in Fig. 5b and c, the percentage of $\mathrm{Gr}-1$-positive cells significantly increased after CLP, and they partially expressed iNOS in the iNOSKO BMT CLP group. In contrast, the number of Gr-1positive cells was not significantly different between the WT BMT CLP group and iNOS-KO BMT CLP group, whereas that of CD11b-positive cells decreased significantly in the iNOS-KO BMT group than in the WT BMT CLP group. This suggests that the decrease in the number of CD11b-positive cells or other populations in the iNOS-KO BMT group may be related to the relative increase in the percentage of Gr-1-positive cells after CLP.

In summary, we established an iNOS-dependent sepsis model via modified keyhole-type CLP, and prevention of iNOS-improved mortality and hypothermia in the late phase of sepsis, which was significantly related to iNOS-derived NO. Sustained hypothermia may indicate overproduction of NO from iNOS and evolution of septic shock, and may be a beneficial guide for future iNOS-targeted therapies. Moreover, iNOS was involved in the pathology of septic ARDS, and modulated the migration and populations of BMDCs. There are few therapies for sepsis at present and we should re-consider iNOS as a potential therapeutic target. In addition, we should develop organ and cell type-specific analyses of iNOS, and validate them in clinical research using selective iNOS inhibitors.

Acknowledgements We would like to thank Dr. Koichi Oishi, a member of Professor Sawada's laboratory, for his technical assistance.

\section{Compliance with ethical standards}

Conflict of interest The authors declare that they have no conflict of interest.

\section{References}

1. Singer M, Deutschman CS, Seymour CW, et al. The Third International Consensus Definitions for Sepsis and Septic Shock (Sepsis-3). JAMA. 2016;315:801-10.

2. Levy MM, Fink MP, Marshall JC, et al. 2001 SCCM/ESICM/ ACCP/ATS/SIS International Sepsis Definitions Conference. Crit Care Med. 2003;31:1250-6.

3. Titheradge MA. Nitric oxide in septic shock. Biochim Biophys Acta. 1999;1411:437-55.

4. Cobb JP, Hotchkiss RS, Swanson PE, et al. Inducible nitric oxide synthase (iNOS) gene deficiency increases the mortality of sepsis in mice. Surgery. 1999;126:438-42.

5. Okamoto I, Abe M, Shibata K, et al. Evaluating the role of inducible nitric oxide synthase using a novel and selective inducible nitric oxide synthase inhibitor in septic lung injury produced by cecal ligation and puncture. Am J Respir Crit Care Med. 2000;162:716-22.

6. Wei XQ, Charles IG, Smith A, et al. Altered immune responses in mice lacking inducible nitric oxide synthase. Nature. 1995;375:408-11.

7. Cuzzocrea S, Mazzon E, Di Paola R, et al. A role for nitric oxidemediated peroxynitrite formation in a model of endotoxin-induced shock. J Pharmacol Exp Ther. 2006;319:73-81.

8. Kushimoto S, Gando S, Saitoh D, et al. The impact of body temperature abnormalities on the disease severity and outcome in patients with severe sepsis: an analysis from a multicenter, prospective survey of severe sepsis. Crit Care. 2013;17:R271. 
9. Laupland KB, Zahar JR, Adrie C, et al. Determinants of temperature abnormalities and influence on outcome of critical illness. Crit Care Med. 2012;40:145-51.

10. Hiller S, DeKroon R, Xu L, et al. alpha-Lipoic acid protects mitochondrial enzymes and attenuates lipopolysaccharide-induced hypothermia in mice. Free Radic Biol Med. 2014;71:362-7.

11. Saia RS, Carnio EC. Thermoregulatory role of inducible nitric oxide synthase in lipopolysaccharide-induced hypothermia. Life Sci. 2006;79:1473-8.

12. Nathan C. Inducible nitric oxide synthase: what difference does it make? J Clin Invest. 1997;100:2417-23.

13. Varisco BM. The pharmacology of acute lung injury in sepsis. Adv Pharmacol Sci. 2011;2011:254619.

14. Mehta S. The effects of nitric oxide in acute lung injury. Vasc Pharmacol. 2005;43:390-403.

15. Razavi HM, Wang L, Weicker S, et al. Pulmonary oxidant stress in murine sepsis is due to inflammatory cell nitric oxide*. Crit Care Med. 2005;33:1333-9.

16. Wang le F, Patel M, Razavi HM, et al. Role of inducible nitric oxide synthase in pulmonary microvascular protein leak in murine sepsis. Am J Respir Crit Care Med. 2002;165:1634-9.

17. Wang LF, Mehta S, Weicker S, et al. Relative contribution of hemopoietic and pulmonary parenchymal cells to lung inducible nitric oxide synthase (inos) activity in murine endotoxemia. Biochem Biophys Res Commun. 2001;283:694-9.

18. Rafat N, Dacho C, Kowanetz G, et al. Bone marrow-derived progenitor cells attenuate inflammation in lipopolysaccharideinduced acute respiratory distress syndrome. BMC Res Notes. 2014;7:613

19. Yamada M, Kubo H, Kobayashi S, et al. Bone marrow-derived progenitor cells are important for lung repair after lipopolysaccharide-induced lung injury. J Immunol. 2004;172:1266-72.

20. Gupta N, Su X, Popov B, et al. Intrapulmonary delivery of bone marrow-derived mesenchymal stem cells improves survival and attenuates endotoxin-induced acute lung injury in mice. J Immunol. 2007; 179:1855-63.

21. Buras JA, Holzmann B, Sitkovsky M. Animal models of sepsis: setting the stage. Nat Rev Drug Discov. 2005;4:854-65.

22. Wichterman KA, Baue AE, Chaudry IH. Sepsis and septic shock - a review of laboratory models and a proposal. J Surg Res. 1980;29:189-201.

23. Matsuda N, Yamamoto S, Yokoo H, et al. Nuclear factor-kappaB decoy oligodeoxynucleotides ameliorate impaired glucose tolerance and insulin resistance in mice with cecal ligation and puncture-induced sepsis. Crit Care Med. 2009;37:2791-9.

24. Matsuda N, Hattori Y, Jesmin S, et al. Nuclear factor-kappaB decoy oligodeoxynucleotides prevent acute lung injury in mice with cecal ligation and puncture-induced sepsis. Mol Pharmacol. 2005;67:1018-25.

25. Ono K, Takii T, Onozaki K, et al. Migration of exogenous immature hematopoietic cells into adult mouse brain parenchyma under GFP-expressing bone marrow chimera. Biochem Biophys Res Commun. 1999;262:610-4.

26. Ono K, Yoshihara K, Suzuki H, et al. Preservation of hematopoietic properties in transplanted bone marrow cells in the brain. $\mathrm{J}$ Neurosci Res. 2003;72:503-7.
27. Okabe M, Ikawa M, Kominami K, et al. 'Green mice' as a source of ubiquitous green cells. FEBS Lett. 1997;407:313-9.

28. Suzuki H, Ono K, Sawada M. Protective effect of INI-0602, a gap junction inhibitor, on dopaminergic neurodegeneration of mice with unilateral 6-hydroxydopamine injection. J Neural Transm. 2014;121:1349-55.

29. Aggarwal NR, King LS, D'Alessio FR. Diverse macrophage populations mediate acute lung inflammation and resolution. Am J Physiol Lung Cell Mol Physiol. 2014;306:L709-25.

30. Kanda Y. Investigation of the freely available easy-to-use software 'EZR' for medical statistics. Bone Marrow Transplant. 2013;48:452-8.

31. Leenen PJ, de Bruijn MF, Voerman JS, et al. Markers of mouse macrophage development detected by monoclonal antibodies. J Immunol Methods. 1994;174:5-19.

32. Ling V, Luxenberg D, Wang J, et al. Structural identification of the hematopoietic progenitor antigen ER-MP12 as the vascular endothelial adhesion molecule PECAM-1 (CD31). Eur J Immunol. 1997;27:509-14.

33. Matsuda N, Hattori Y, Takahashi Y, et al. Therapeutic effect of in vivo transfection of transcription factor decoy to NF-kappaB on septic lung in mice. Am J Physiol Lung Cell Mol Physiol. 2004;287:L1248-55.

34. Inoue S, Shinjo T, Kawaguchi M, et al. Amino acid infusions started after development of intraoperative core hypothermia do not affect rewarming but reduce the incidence of postoperative shivering during major abdominal surgery: a randomized trial. J Anesth. 2011;25:850-4.

35. Fonseca MT, Rodrigues AC, Cezar LC, et al. Spontaneous hypothermia in human sepsis is a transient, self-limiting, and nonterminal response. J Appl Physiol. 2016;120:1394-401.

36. Liu HW, Anand A, Bloch K, et al. Expression of inducible nitric oxide synthase by macrophages in rat lung. Am J Respir Crit Care Med. 1997;156:223-8.

37. Kobayashi H, Hataishi R, Mitsufuji H, et al. Antiinflammatory properties of inducible nitric oxide synthase in acute hyperoxic lung injury. Am J Respir Cell Mol Biol. 2001;24:390-7.

38. Sonego F, Castanheira FV, Ferreira RG, et al. Paradoxical roles of the neutrophil in sepsis: protective and deleterious. Front Immunol. 2016;7:155.

39. Lerman YV, Kim M. Neutrophil migration under normal and sepsis conditions. Cardiovase Hematol Disord Drug Targets. 2015;15:19-28.

40. Gabrilovich DI, Nagaraj S. Myeloid-derived suppressor cells as regulators of the immune system. Nat Rev Immunol. 2009;9:162-74.

41. Ost M, Singh A, Peschel A, et al. Myeloid-derived suppressor cells in bacterial infections. Front Cell Infect Microbiol. 2016;6:37.

42. Cuenca AG, Moldawer LL. Myeloid-derived suppressor cells in sepsis: friend or foe? Intensive Care Med. 2012;38:928-30.

43. Satoh T, Nakagawa K, Sugihara F, et al. Identification of an atypical monocyte and committed progenitor involved in fibrosis. Nature. 2017;541:96-101.

44. Zhang Y, Wittner M, Bouamar H, et al. Identification of CXCR4 as a new nitric oxide-regulated gene in human CD34+ cells. Stem Cells. 2007;25:211-9. 